\title{
Water and lysozyme: Some results from the bending and stretching vibrational modes
}

\author{
Francesco Mallamace ${ }^{1,2,3,4, \dagger}$, Carmelo Corsaro ${ }^{1,4}$, Domenico Mallamace $^{5}$, Cirino Vasi ${ }^{4}$, \\ ${\text { Nicola } \text { Cicero }^{5}, \text { H. Eugene Stanley }}^{3}$ \\ ${ }^{1}$ Dipartimento di Fisica e Scienze della Terra, Università di Messina, I-98166, Messina, Italy \\ ${ }^{2}$ Department of Nuclear Science and Engineering, Massachusetts Institute of Technology, Cambridge, MA 02139, USA \\ ${ }^{3}$ Center for Polymer Studies and Department of Physics, Boston University, Boston, MA 02215, USA \\ ${ }^{4}$ Consiglio Nazionale delle Ricerche-IPCF Messina, I-98166, Messina, Italy \\ ${ }^{5}$ Dipartimento di Scienze dell'Ambiente, della Sicurezza, del Territorio, degli Alimenti, e della Salute, \\ Università di Messina, Viale F. Stagno d'Alcontres 31, 98166 Messina, Italy \\ Corresponding author. E-mail: †francesco.mallamace@unime.it \\ Received July 7, 2015; accepted August 24, 2015
}

\begin{abstract}
The dynamic or glass transition in biomolecules is important to their functioning. Also essential is the transition between the protein native state and the unfolding process. To better understand these transitions, we use Fourier transform infrared spectroscopy to study the vibrational bending and stretching modes of hydrated lysozymes across a wide temperature range. We find that these transitions are triggered by the strong hydrogen bond coupling between the protein and hydration water. More precisely, we demonstrate that in both cases the water properties dominate the evolution of the system. We find that two characteristic temperatures are relevant: in the supercooled regime of confined water, the fragile-to-strong dynamic transition occurs at $T_{L}$, and in the stable liquid phase, $T^{*} \simeq 315 \pm 5 \mathrm{~K}$ characterizes the behavior of both isothermal compressibility $K_{T}(T, P)$ and the coefficient of thermal expansion $a_{P}(T, P)$.
\end{abstract}

Keywords protein unfolding, hydration water, infrared spectroscopy

PACS numbers 87.64.km, 87.15.kr, 82.30.Rs

\section{Introduction}

Although water is a simple molecule, it has intriguing and counterintuitive physicochemical behaviors that have not been clearly explained [1]. The enormous number of studies on water have all come to the same conclusion: hydrogen bond (HB) interactions between water molecules are the key to understanding its properties and functioning [2], especially in biological environments [3]. As the temperature $T$ decreases, the HBs cluster and form an open tetrahedrally coordinated HB network. This HB clustering explains such thermodynamic anomalies as the density maximum at $4^{\circ} \mathrm{C}$ and the diverging behavior of various thermal response functions when the temperature is decreased into the supercooled region of the phase diagram. More precisely, when the temperature of the stable liquid phase is lowered, both the HB lifetime and cluster stability increase, and this altered local structure can, in principle, continue down to the amorphous region of the phase diagram.

Water is not a simple solvent in biosystems. It controls their stability, dynamics, and function [4] and takes an active role in, e.g., the protein folding-unfolding process and the life of cells [5]. Understanding the contribution of HBs to protein stability and to the structural flexibility and dynamics of macromolecules enables us to understand the complex properties of water and their influence on the behavior of biological systems.

Below $T_{g} \approx 130 \mathrm{~K}$, water is a glass. Above that temperature, it becomes a highly viscous fluid that crystallizes at $T_{X} \approx 150 \mathrm{~K}$. Amorphous water, like ice, is polymorphic. The two phases of glassy amorphous water, low density (LDA) and high density (HDA), can be transformed from one to the other by tuning the pressure. Metastable supercooled water is located on 
the phase diagram between the homogeneous nucleation temperature, $T_{H}=231 \mathrm{~K}$, and the melting temperature, $T_{M}=273 \mathrm{~K}$. The region between $T_{X}$ and $T_{H}$ is not experimentally accessible for bulk liquid water and is referred to as the "no man's land" of the phase diagram, but crystallization within this region can be retarded somewhat by confining the water within narrow nanoporous structures or on a protein surface.

The polymorphism of glassy water and the HB networking suggest that liquid water may also be polymorphous, i.e., a mixture of a low-density liquid (LDL) and a high-density liquid (HDL) that predominates in the high$T$ regime. In the HDL phase, the local tetrahedrally coordinated HB structure is not fully developed, but in the LDL, a more open ice-like HB network appears. Thus, water's anomalies are caused by the competition between these two local liquid forms. Studies of confined water $[6,7]$ have found that decreasing $T$ greatly increases the water HB lifetime, indicating the presence of a dynamic crossover from a super-Arrhenius (fragile) to an Arrhenius (strong) liquid at $T_{L} \simeq 225 \mathrm{~K}$ and ambient pressure. At this temperature, the Stokes-Einstein relation is violated $[6,8]$, and clear signs of LDL and HDL are observed [7], suggesting that polymorphism also exists in the liquid phase.

This fragile-to-strong dynamic crossover (FSDC), with $T_{L}>T_{g}$, is not limited to water but also characterizes all supercooled glass-forming liquids [9]. Its appearance indicates that the system is approaching dynamic arrest [917], and many special processes occur at that point (see, e.g., Ref. [12]), including violation of the Stokes-Einstein law, orientational-translational decoupling [18], splitting of the relaxation into primary $(\alpha)$ and secondary relaxation times $(\beta)$, the onset of dynamical heterogeneities, and the onset of the boson peak (hypothesized for bulk water [15] and observed in confined water [16]).

The FSDC is highly relevant in biology because it is related to the dynamic transition that biomolecules undergo in the low- $T$ regime $[17,19-23]$. At the lowest temperatures, proteins are in a glassy state, a solid-like structure without conformational flexibility [24]. When $T$ is increased, measurements of the mean-squared atomic displacement (MSD), $\left\langle X^{2}\right\rangle$, reveal that the atomic motional amplitude increases linearly, as in a harmonic solid. In hydrated proteins, its rate suddenly increases at $\sim 220 \mathrm{~K}$, signaling the onset of an additional anharmonic and liquid-like motion $[25,26]$. The functions and kinetics of the biochemical reactions in many proteins slow down sharply at a temperature $T_{C}$ in a universal range of 240-200 K [27]. This transition can be suppressed in dry biomolecules [26], and the protein biochemical activity depends on their level of hydration $h$ (i.e., grams of
$\mathrm{H}_{2} \mathrm{O}$ per grams of dry protein). For lysozyme, $h=0.3$ corresponds to a water monolayer covering the protein surface; the enzymatic activity is very low up to a hydration level of 0.2 and then increases sharply as $h$ increases from 0.2 to 0.5 [28].

In a protein-water solution, water can influence both the hydrophilic and hydrophobic side groups of a biomolecule, and in this respect two water categories are of interest: bound internal water and surface (or hydration) water. The first layer of water, in strong interaction with the protein surface, is the hydration water. The bound internal water molecules, located in internal cavities and clefts, are extensively involved in proteinsolvent $\mathrm{H}$ bonding and play a structural role in the folded protein itself. The hydration water, interacting with the solvent-exposed protein atoms having different chemical character, feels the topology and roughness of the protein surface and is believed to have an important role in controlling the biofunctionality of the protein $[29,30]$.

The study of the physical properties of confined water depending on the cavity surface and/or the confinement dimensions is a very active field. Indeed, water structures and dynamics in confined environments are studied to improve knowledge of geological and biological processes. However, despite the numerous experimental and theoretical studies, the behavior of water at hydrophilic and hydrophobic interfaces is still not completely understood $[31,32]$.

Hydrophilicity (which results from the HB strength) is a force that governs the secondary structure and folding specificity of proteins [33], but the properties of the biomolecule can also be affected by the protein methyl groups. It has been shown that although a single hydration water layer can influence both the hydrophilic chains and the dynamics of the entire protein, the dynamic effects on the methyl chains are minimal (their motions are confined and attributed to librational and rotational movements). Several hydration shells of water are required for the hydrophobic side chains to exhibit, at room temperature, the full range of motions characteristic of a liquid-like protein state [34]. Hence, because the properties of the hydration water are intimately connected to protein stability and function, an understanding of the hydrophilic interactions with peptide groups is essential for the study of biological systems.

The approximate coincidence of $T_{L}$ (the water dynamic crossover temperature) and $T_{C}$ (at which biochemical activities slow and $\left\langle X^{2}\right\rangle$ rises sharply in biomolecules) has suggested a connection between the two phenomena. Specifically, this dynamic transition in proteins is believed to be triggered by their strong $\mathrm{HB}$ coupling with the hydration water [35]. This behavior has 
been observed in several biomolecules, including globular proteins [19, 20], DNA, and RNA [21].

This dynamic crossover in protein water has been studied by measuring the MSD and the transport parameters — the self-diffusion coefficient $D_{S}$ and the average translational relaxation time $\langle\tau\rangle$ as a function of $T-$ using various experimental techniques ranging from neutron scattering [19] to nuclear magnetic resonance (NMR) [20].

The FSDC can be observed in water only under confinement, but there is another transition that characterizes thermodynamically stable bulk water. Recent studies of water density as a function of both temperature and pressure [36-38] propose the existence of a "special" temperature, $T^{*} \simeq 315 \pm 5 \mathrm{~K}$, that characterizes the behavior of both the isothermal compressibility $K_{T}(T, P)$ and the coefficient of thermal expansion $a_{P}(T, P)[39,40]$. More precisely, $K_{T}(T, P)$ shows a minimum for all the studied pressures at $T^{*}$, and all the $\alpha_{P}(T)=1 / V(\partial V / \partial T)_{P}$ curves, measured at different pressures $P$, cross at a "singular and universal expansivity point" located at exactly the same temperature, $T^{*}$ $\left(a_{P}\left(T^{*}\right) \sim 0.44 \times 10^{-3} \mathrm{~K}^{-1}\right)$. This temperature marks the curvature of $\alpha_{P}(T)$. Unlike other water singularities, this temperature is thermodynamically consistent with the relationship connecting the two response functions. Analysis of transport parameters such as the viscosity and the self-diffusion coefficient $D_{S}$ has revealed that decreasing the temperature to $T^{*}$ causes decoupling of the roto-translational dynamics [41] and a crossover from strong to fragile dynamics to occur (the opposite of what happens at $T_{L}$ ) [40]. The $P-T$ behavior of the selfdiffusion coefficient $D_{S}$ and of the NMR proton chemical shift $\delta$ indicates that if we decrease $T$, at $T^{*}$ a crossover from the normal fluid to the anomalous fluid occurs, and we have a complex liquid characterized by many anomalies.

In this work, we study the Fourier transform infrared (FTIR) spectra to verify how these two temperatures, $T_{L}$ and $T^{*}$, which characterize confined water in the supercooled state and bulk water in the stable phase, respectively, can influence protein dynamics.

FTIR spectroscopy is among the experimental techniques most suitable for studying the structure and dynamics of proteins in aqueous solutions [42]. In fact, the different amide bands found in IR spectra (see the Results and Discussion) provide information about the secondary structure of proteins, which depends on the protein configuration. Their study, together with that of the water bands, can shed light on the mechanisms that govern the system's thermodynamic properties.

\section{Experiments}

We study the globular protein lysozyme hydrated with a single monolayer of water. Samples are prepared by hydrating dried protein powder isopiestically at $5^{\circ} \mathrm{C}$ by exposing it to water vapor in a closed chamber until the desired hydration level $h$ is reached (here we use $h=0.3$ and 0.37 ). We use differential scanning calorimetry to test for the absence of bulk-like water.

We take FTIR absorption measurements at ambient pressure using a PerkinElmer Spectrum GX Fourier transform spectrometer with an attenuated total reflection geometry. We record the measured spectra at a resolution of $4 \mathrm{~cm}^{-1}$ by performing 250 repetitive scans in order to obtain a high reproducibility and then normalize them. We initially cool the samples to $180 \mathrm{~K}$ and then measure them while increasing $T$ in $10 \mathrm{~K}$ steps to $350 \mathrm{~K}$, i.e., from below the protein dynamic crossover temperature in the protein native state to above the irreversible denaturation temperature $\left(T_{D} \approx 345 \mathrm{~K}\right)$. The temperature increase is slow, and its stability is maintained in a range of $0.1 \mathrm{~K}$.

\section{Results and discussion}

Figure 1 shows the vibrational bending mode (measured at $300 \mathrm{~K}$ ) of pure bulk water (upper panel) and the peptide modes (amide I, amide II) falling in the spectral region $1300 \mathrm{~cm}^{-1}<\nu<1800 \mathrm{~cm}^{-1}$ (lower panel). The peptide groups are described using nine characteristics bands in the IR and Raman spectra; the bands are designated amide A, B, and I-VII in order of decreasing frequency. Amides I and II are the two major bands of the protein IR spectrum. Amides III and IV, resulting from a mixture of several coordinate displacements, are very complex. Out-of-plane motions are found in amides V, VI, and VII. Amides I, II, and III are used to assign the protein secondary structure [44] and fall within the frequency range in which the water bending modes are located (1300-1800 $\left.\mathrm{cm}^{-1}\right)$. Water has two bending modes, one caused by molecules clustered in the network (i.e., only the four-bonded or LDL, at $\sim 1560 \mathrm{~cm}^{-1}$ ), and a second due to the remaining, nonclustered free network molecules (the HDL, at $\sim 1640 \mathrm{~cm}^{-1}$ ) [43]. Amide I, which is determined by the peptide backbone conformation (and is independent of the amino acid sequence, its hydrophilic or hydrophobic properties, and its charge), contributes (i) the $\alpha$ helix $\left(1650-1657 \mathrm{~cm}^{-1}\right)$, (ii) the antiparallel $\beta$ sheets (two contributions in the ranges 1612 $1640 \mathrm{~cm}^{-1}$ and $1680-1689 \mathrm{~cm}^{-1}$ ), and (iii) the random 


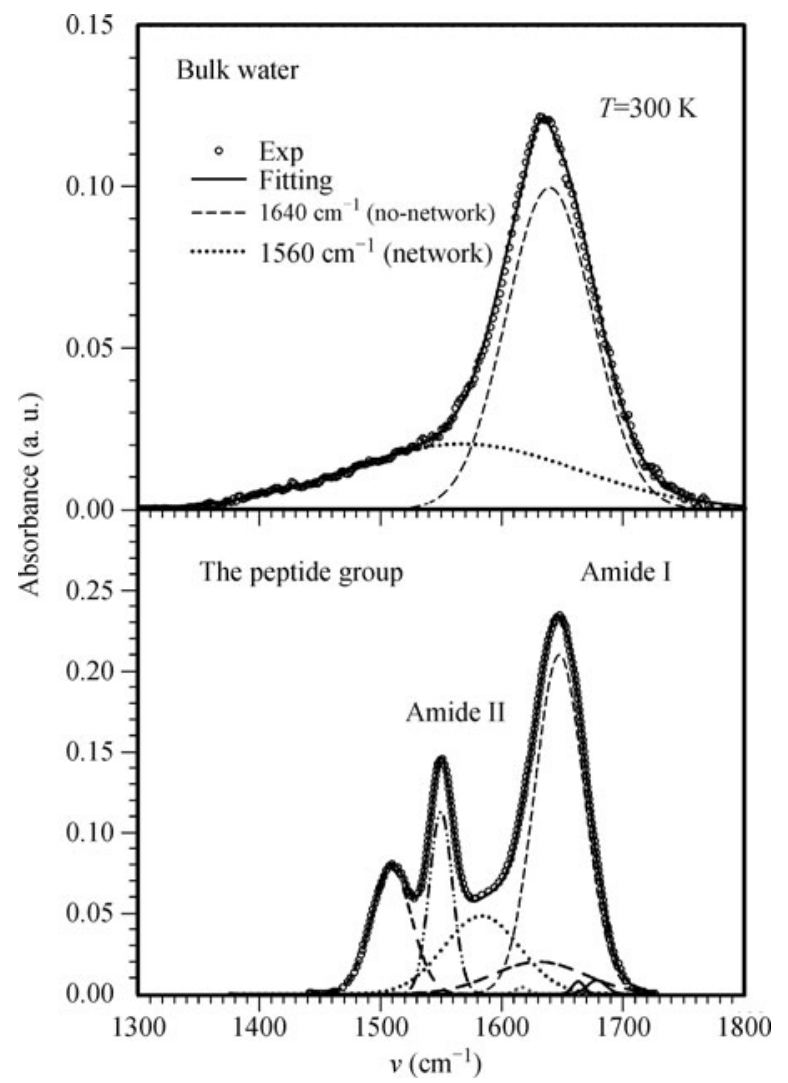

Fig. 1 The bending spectrum of pure bulk water at $300 \mathrm{~K}$ (top) in the range $1300 \mathrm{~cm}^{-1}<\nu<1800 \mathrm{~cm}^{-1}$. The peptide groups, Amide I, Amide II, falling in the same spectral range (bottom panel). Reproduced from Ref. [5].

coil near $1680 \mathrm{~cm}^{-1}$. The denatured proteins in the native state exhibit highly differentiated spectra [45]. There is a process characterizing the unfolding in the amide II band, and the amide II $N-H$ residual disappears (1530$\left.1550 \mathrm{~cm}^{-1}\right)$.

Amide I absorption is caused by $C=O$ stretching vibration, and amide II absorption is due to $N-H$ bending vibration and $C-N$ stretching vibration at $1540 \mathrm{~cm}^{-1}$ and $1520 \mathrm{~cm}^{-1}$, respectively. Using this information, a study of the bending vibrational mode of water and protein reveals that the HBs in water molecules with a protein peptide carbonyl oxygen $(\mathrm{C}=\mathrm{O})$ and an amide $(\mathrm{N}-$ $H)$ molecular group trigger the biomolecular dynamic transition $[5,46,47]$. The most stable configuration has two HBs, a water proton donor bond to the carbonyl oxygen and an amide $N-H$ proton donor bond to the water oxygen $[46,47]$. Water acts as an HB "glue" between the protein's carbonylic and amidic groups [48] by governing the protein folding and secondary structure. All of this affects both the frequency and the extinction coefficient of IR absorption lines because the normal mode force constant is modified.

Figure 2 shows the vibrational $\mathrm{OH}$ stretching spectra $\left(2700 \mathrm{~cm}^{-1}<\nu<3700 \mathrm{~cm}^{-1}\right)$ of the water-lysozyme system with a hydration level of $h=0.3$ at several different temperatures in the range $180 \mathrm{~K}<T<350 \mathrm{~K}$. For comparison, it also shows the corresponding spectrum of pure bulk water measured at $293 \mathrm{~K}$. Note that there is a marked difference between the bulk water data (blue circles) and hydrated protein data (blue line) at the same temperature. There is a large red-shift in the central $\mathrm{O}-\mathrm{H}$ stretching frequency of protein hydration water $\left(\sim 50 \mathrm{~cm}^{-1}\right)$ relative to the bulk. The shift increases toward lower temperatures and is characterized by a marked increase in the intensity of the hydration water in the region of the lowest frequencies, which is consistent with the contraction and increasing strength of the protein-water bonds; i.e., there is also a marked increase in the population of the bonded water. Unlike bulk water molecules, most water hydration molecules are bonded (with the protein hydrophilic group or in a water-water HB) [2]. Note that the spectra in Figs. 1 and 2 (where the protein-water vibrational dynamics change significantly with $T$ ) differ completely from those of crystalline and amorphous ice, indicating that the water is in a liquid phase.

The study of water and water solutions has traditionally focused on molecular stretching modes that are easily accessible using standard Raman and IR spectroscopic techniques because of their high absorption cross sections and frequencies. The relaxation of vibrational excitations in water reflects its physical properties [44]. The bending modes also exhibit analogous features because they are sensitive to the HB frequency, HB strength, and water molecule connectivity.

Bending mode relaxation differs from stretching be-

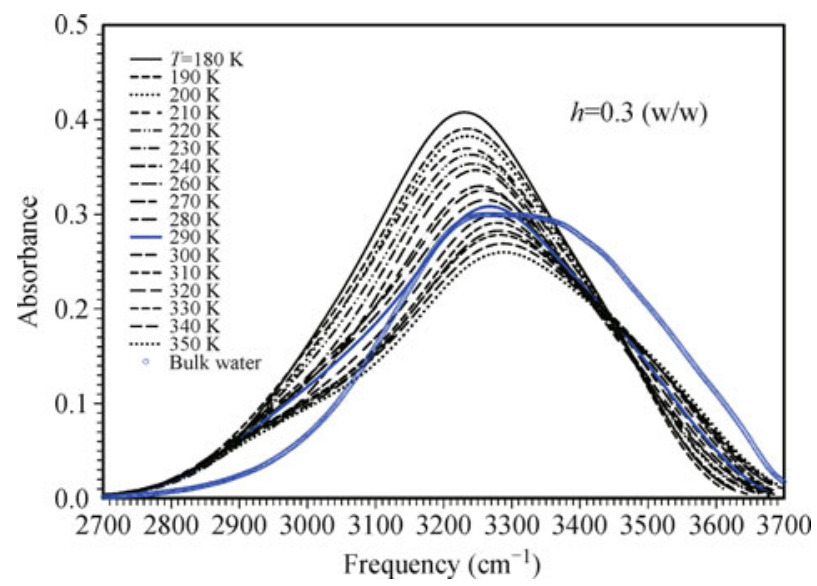

Fig. 2 The measured FTIR vibrational $\mathrm{OH}$-stretching spectra $\left(2700 \mathrm{~cm}^{-1}<\nu<3700 \mathrm{~cm}^{-1}\right)$ of the water lysozyme system with an hydration level of $h=0.3$, at several different temperatures in the range $180 \mathrm{~K}<T<350 \mathrm{~K}$; for comparison the corresponding spectrum of the pure bulk water measured at $293 \mathrm{~K}$ is also reported. 
cause it occurs through intermolecular interactions and consequent energy transfers (stretching is essentially an intramolecular mode). These couplings occur in pure systems and in solutions, with the difference that in solutions these intermolecular interactions involve an energy transfer between solute and solvent (i.e., protein and water) [51].

Figure 1 shows the water contribution to the amide 1 bending mode. Walrafen et al. [44] use this spectral component to determine the spectral contributions of water inside (LDL) and outside (HDL) the network; the corresponding data can be used to evaluate the enthalpy value $H$ of the HB formation process. For this, the van't Hoff expression, which relates the $T$ dependence of an equilibrium constant $K$ to the change in $H$ as $\mathrm{d} \ln K / \mathrm{d}(1 / T)=-\Delta H / R$, can be used. We extracted these two contributions using a spectral deconvolution with Gaussian functions [42, 44]. Figure 3 shows a loglinear plot of the ratio $A_{N E T} / A_{N C}$ of the integrated areas of the fully tetrabonded molecules $\left(1560 \mathrm{~cm}^{-1}\right)$ and the molecules outside the network and not clusterized $\left(1640 \mathrm{~cm}^{-1}\right)$ versus $1 / T$. To clarify, we assume that the ratio $A_{N E T} / A_{N C}$ is directly related to $K$, and, from $\ln \left(A_{N E T} / A_{N C}\right)=-\Delta H / R T$, we obtain the HB enthalpy variation in the large $T$ interval under study. We examine data for bulk water (from experiments and from Ref. [44]) and confined water (in MCM nanotubes [50] and lysozyme at $h=0.3$ and 0.37 ).

Figure 3 shows the results and confirms that there are different regimes immediately above and below the main temperatures characterizing the HBs, $T_{L}$ and $T^{*}$, of the system. When we decrease $T$ below the high-temperature regime of stable bulk water, the ratio first shows a flat minimum near $T^{*}$ and then increases linearly to the

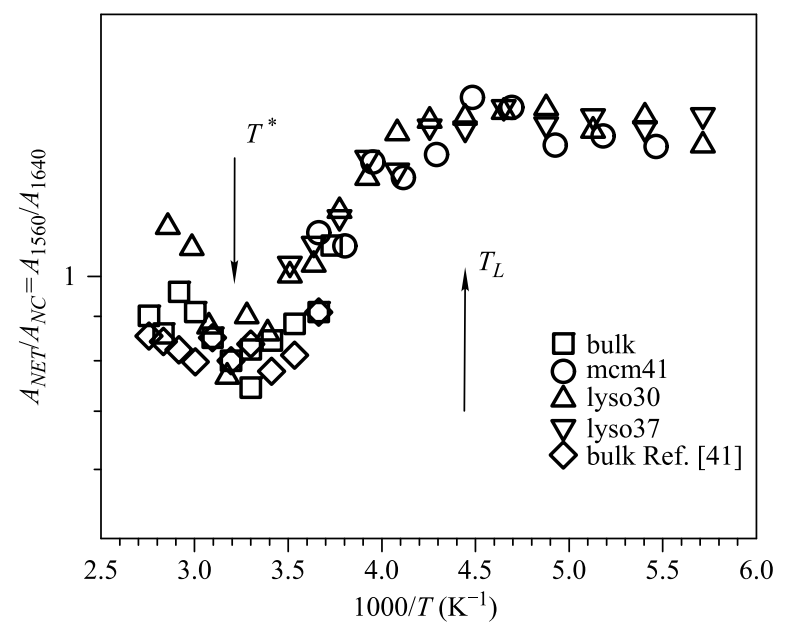

Fig. 3 The ratio $A_{N E T} / A_{N C}$ on a logarithmic scale versus 1000/T (180 K $<T<365 \mathrm{~K})$. Data of bulk (experiments and Ref. [44]) and confined water (in MCM nanotubes [50] and lysozyme at $h=0.3$ and 0.37$)$ are reported.
FSDC temperature $\left(T_{L}\right)$. Below the crossover temperature, it remains constant. This confirms the physical roles of $T^{*}$ and $T_{L}$. At $T^{*}$, a tetrabonded water cluster is created, and at the FSDC (the onset of the protein harmonic solid-like state), a majority of the water molecules are linked to either the tetrabonded water cluster (the network or LDL phase) or to the protein by $\mathrm{C}=\mathrm{O}-\mathrm{HO}$ and $\mathrm{NH}-\mathrm{O}$ bonds. The probability that an $\mathrm{HB}$ will form or break is low, and the only water motion is caused by hopping between clusters. Increasing $T$ allows HBs in the anharmonic protein liquid phase to be formed and broken, and the corresponding probability evolves with $T$ according to the van't Hoff equation. The enthalpy change $\Delta H$ of the water HB can be evaluated (i.e., $\sim 2.3$ $\mathrm{kcal} / \mathrm{mol}$, typical of HBs) by plotting between these two main temperatures.

Finally, we quantitatively analyze the red-shift in the central frequency of $\mathrm{OH}$ stretching of protein hydration water (see Fig. 2), which may be the molecular mechanism underlying two dynamic transitions: (i) the transition originally observed in neutron scattering of the MSD of the hydrated protein, indicating the onset of an additional anharmonic liquid-like motion $[25,26]$ strongly related to the dynamical crossover, and (ii) the transition at the onset of the stable tetrahedral HB structure that characterizes water and its thermodynamic anomalies. Figure 4 shows the $\mathrm{OH}$ stretching frequencies of hydrated lysozyme at hydration levels of $h=0.3$ and 0.37 as a function of the temperature in the interval $180 \mathrm{~K}<T<365 \mathrm{~K}$. We increase the temperature and observe three linear behaviors: one for $T<T_{L}$, one in the interval between $T_{L}$ and $T^{*}$, and one for $T>T^{*}$. These behaviors highlight the importance of both $T_{L}$ and $T^{*}$ and indicate how, because of the HBs between the water and the water-protein molecules, the dynamics of water vibration change the structure of the hydration water. This is caused by the correlation between the temperature dependence of the $\mathrm{O}-\mathrm{H}$ stretching frequency and

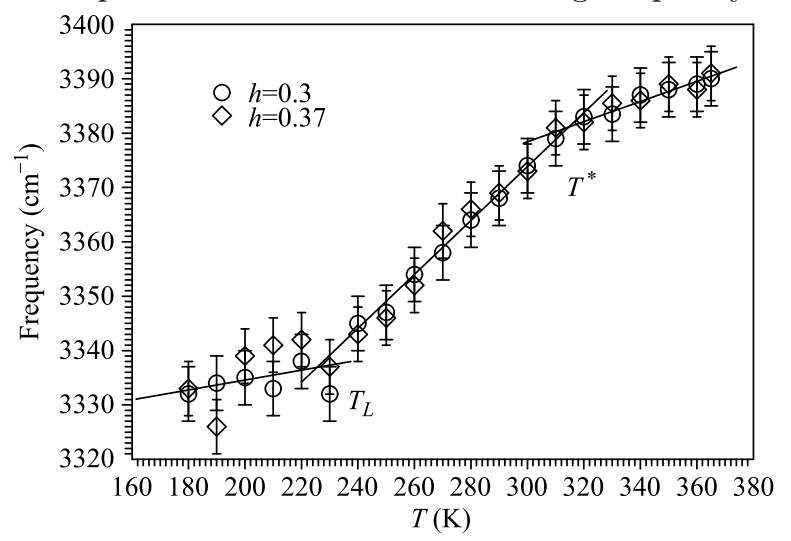

Fig. 4 The frequency behaviors of the protein water $\mathrm{OH}$ stretching contibution illustrated as a function of the temperature $T$. 
the thermal expansion characteristic of hydrogen-bonded systems [52].

In structured (i.e., quasi-harmonic solid or solidlike) systems, the thermal expansion $\alpha_{P}(T)$ is linked to the normal mode frequencies $\nu(n)$ via the so-called Grüneisen parameters of mode $\gamma(n)$. In particular, $\nu(n)$ decreases as the volume increases $\left(\nu(n) \propto V^{-\gamma(n)}\right)$. Thus, the relative changes of the mode frequencies with $T$ are determined by the volume expansion coefficient $(-\partial \nu(n) / \partial T) \sim(\gamma(n) \nu(n)) \alpha(T))$. Because a quantitative correlation between the $\mathrm{OH}$ stretching frequency and the $\mathrm{O}-\mathrm{O}$ separation has been observed in ice I [52], we assume that it holds also for water in tetrabonded structures and LDL clusters. The force constants in this structured water depend on the strength of the HB, which affects the $\mathrm{OH}$ stretching vibration. Thus, the $\mathrm{OH}$ stretching frequency increases with $T$, and the thermal expansion (the increase in the lattice parameters) reflects the behavior of a "linear Grüneisen relation" $[52,53]$ and generates new HB configurations (i.e., changes the structure). Figure 2 shows this behavior above and below $T_{L}$, where the structural change in confined water is accompanied by the protein dynamic transition. Although this clarifies $T^{*}$ and shows it to be the temperature at which the curvature of bulk water $\alpha_{P}(T)$ changes, the frequency change around $T^{*}$ is even more interesting [39, 40]. Recent NMR experiments focusing on the protein hydrophilic (amide $\mathrm{NH}$ ) group, the hydrophobic (methyl $\left.\mathrm{CH}_{3}\right)$ group, and methine $(\mathrm{CH})$ indicate that $T^{*}$ may represent the high-temperature limit of the protein native state [54].

\section{Conclusion}

In conclusion, our findings focus on how HB networking in water affects protein hydrophilic groups. The hydrophobic groups that appear when the protein is in its native folded state (i.e., up to $T^{*}$ ) are buried within the protein interior [4]. In the wide temperature region spanning the protein glass state to the onset of unfolding, the interaction between water and the carbonylic and amidic groups of the protein plays an important role together with the HB network of the hydration water [48]. This is the basis of protein folding and its secondary structure, and also of the two crossovers observed in the bending and stretching modes of the hydrated lysozyme. Below $T^{*}$, water cross-links with the protein hydrophilic groups, and above $T^{*}$, the HBs become weaker and allow the protein to unfold [38, 40].

Open Access This article is distributed under the terms of the
Creative Commons Attribution License which permits any use, distribution, and reproduction in any medium, provided the original author(s) and the source are credited.

\section{References and notes}

1. P. G. Debenedetti and H. E. Stanley, Supercooled and glassy water, Phys. Today 56(6), 40 (2003)

2. F. Mallamace, P. Baglioni, C. Corsaro, J. Spooren, H. E. Stanley, and S. H. Chen, Transport properties of supercooled confined water, Riv. Nuovo Cim. 34, 253 (2011)

3. G. A. Jeffrey and W. Saenger, Hydrogen Bonding in Biological Structures, Berlin: Springer-Verlag, 1991

4. Y. Levy and J. N. Onuchic, Water mediation in protein folding and molecular recognition, Annu. Rev. Biophys. Biomol. Struct. 35(1), 389 (2006)

5. F. Mallamace, P. Baglioni, C. Corsaro, S. H. Chen, D. Mallamace, C. Vasi, and H. E. Stanley, The influence of water on protein properties, J. Chem. Phys. 141(16), 165104 (2014)

6. S. H. Chen, F. Mallamace, C. Y. Mou, M. Broccio, C. Corsaro, A. Faraone, and L. Liu, The violation of the StokesEinstein relation in supercooled water, Proc. Natl. Acad. Sci. USA 103(35), 12974 (2006)

7. F. Mallamace, M. Broccio, C. Corsaro, A. Faraone, D. Majolino, V. Venuti, L. Liu, C. Y. Mou, and S. H. Chen, Evidence of the existence of the low-density liquid phase in supercooled, confined water, Proc. Natl. Acad. Sci. USA 104(2), 424 (2007)

8. L. Xu, F. Mallamace, Z. Yan, F. W. Starr, S. V. Buldyrev, and H. E. Stanley, Appearance of a fractional StokesEinstein relation in water and a structural interpretation of its onset, Nat. Phys. 5(8), 565 (2009)

9. F. Mallamace, C. Branca, C. Corsaro, N. Leone, J. Spooren, S. H. Chen, and H. E. Stanley, Transport properties of glassforming liquids suggest that dynamic crossover temperature is as important as the glass transition temperature, Proc. Natl. Acad. Sci. USA 107(52), 22457 (2010)

10. S. Yip and M. P. Short, Multiscale materials modeling at the mesoscale, Nat. Mater. 12(9), 774 (2013)

11. F. Mallamace, C. Corsaro, H. E. Stanley, and S. H. Chen, The role of the dynamic crossover temperature and the arrest in glass-forming fluids, Eur. Phys. J. E 34(9), 94 (2011)

12. J. C. Martinez-Garcia, J. Martinez-Garcia, S. J. Rzoska, and J. Hulliger, The new insight into dynamic crossover in glass forming liquids from the apparent enthalpy analysis, $J$. Chem. Phys. 137(6), 064501 (2012)

13. J. C. Mauro, Y. Yue, A. J. Ellison, P. K. Gupta, and D. C. Allan, Viscosity of glass-forming liquids, Proc. Natl. Acad. Sci. USA 106(47), 19780 (2009)

14. K. T. Wikfeldt, A. Nilsson, and L. G. M. Pettersson, Spatially inhomogeneous bimodal inherent structure of simulated liquid water, Phys. Chem. Chem. Phys. 13(44), 19918 (2011) 
15. P. Kumar, K. T. Wikfeldt, D. Schlesinger, L. G. M. Pettersson, and H. E. Stanley, The Boson peak in supercooled water, Sci. Rep. 3, 1980 (2013)

16. S. H. Chen, Y. Zhang, M. Lagi, S. H. Chong, P. Baglioni, and F. Mallamace, Evidence of dynamic crossover phenomena in water and other glass-forming liquids: Experiments, MD simulations and theory, J. Phys.: Condens. Matter 21(50), $504102(2009)$

17. G. Schiró, F. Natali, and A. Cupane, Physical origin of anharmonic dynamics in proteins: New insights from resolution-dependent neutron scattering on homomeric polypeptides, Phys. Rev. Lett. 109(12), 128102 (2012)

18. F. H. Stillinger, A topographic view of supercooled liquids and glass formation, Science 267(5206), 1935 (1995)

19. S. H. Chen, L. Liu, E. Fratini, P. Baglioni, A. Faraone, and E. Mamontov, Observation of fragile-to-strong dynamic crossover in protein hydration water, Proc. Natl. Acad. Sci. USA 103(24), 9012 (2006)

20. X. Q. Chu, A. Faraone, C. Kim, E. Fratini, P. Baglioni, J. B. Leao, and S. H. Chen, Proteins remain soft at lower temperatures under pressure, J. Phys. Chem. B 113(15), 5001 (2009)

21. G. Caliskan, R. M. Briber, D. Thirumalai, V. Garcia-Sakai, S. A. Woodson, and A. P. Sokolov, Dynamic transition in tRNA is solvent induced, J. Am. Chem. Soc. 128(1), 32 (2006)

22. M. Lagi, X. Q. Chu, C. Kim, F. Mallamace, P. Baglioni, and S. H. Chen, The low-temperature dynamic crossover phenomenon in protein hydration water: Simulations vs. experiments, J. Phys. Chem. B 112(6), 1571 (2008)

23. M. Fomina, G. Schirò, and A. Cupane, Hydration dependence of myoglobin dynamics studied with elastic neutron scattering, differential scanning calorimetry and broadband dielectric spectroscopy, Biophys. Chem. 185, 25 (2014)

24. I. E. T. Iben, D. Braunstein, W. Doster, H. Frauenfelder, M. K. Hong, J. B. Johnson, S. Luck, P. Ormos, A. Schulte, P. J. Steinbach, A. H. Xie, and R. D. Young, Glassy behavior of a protein, Phys. Rev. Lett. 62(16), 1916 (1989)

25. F. Parak and E. W. Knapp, A consistent picture of protein dynamics, Proc. Natl. Acad. Sci. USA 81(22), 7088 (1984)

26. G. Zaccai, How soft is a protein? Science 288(5471), 1604 (2000)

27. B. F. Rasmussen, A. M. Stock, D. Ringe, and G. A. Petsko, Crystalline ribonuclease A loses function below the dynamical transition at $220 \mathrm{~K}$, Nature 357(6377), 423 (1992)

28. J. A. Rupley and G. Careri, Protein hydration and function, Adv. Protein Chem. 41, 37 (1991)

29. M. Ferrand, A. J. Dianoux, W. Petry, and G. Zaccai, Thermal motions and function of bacteriorhodopsin in purple membranes: Effects of temperature and hydration studied by neutron scattering, Proc. Natl. Acad. Sci. USA 90(20), 9668 (1993)
30. G. Careri, Cooperative charge fluctuations by migrating protons in globular proteins, Prog. Biophys. Mol. Biol. 70(3), 223 (1998)

31. Q. Li, J. Song, F. Besenbacher, and M. Dong, Twodimensional material confined water, Acc. Chem. Res. 48(1), 119 (2015)

32. S. O. Diallo, E. Mamontov, W. Nobuo, S. Inagaki, and Y. Fukushima, Enhanced translational diffusion of confined water under electric field, Phys. Rev. E 86(2), 021506 (2012)

33. L. Pauling, R. B. Corey, and H. R. Branson, The structure of proteins; two hydrogen-bonded helical configurations of the polypeptide chain, Proc. Natl. Acad. Sci. USA 37(4), 205 (1951)

34. D. Russo, G. L. Hura, and J. R. D. Copley, Effects of hydration water on protein methyl group dynamics in solution, Phys. Rev. E 75(4), 040902 (2007)

35. M. Tarek and D. J. Tobias, Role of protein-water hydrogen bond dynamics in the protein dynamical transition, Phys. Rev. Lett. 88(13), 138101 (2002)

36. P. W. Bridgman, Water, in the liquid and five solid forms, under pressure, Proc. Am. Acad. Arts Sci. 47(13), 441 (1912)

37. T. Grindley and J. E. Lind, PVT properties of water and mercury, J. Chem. Phys. 54(9), 3983 (1971)

38. O. Mishima, Volume of supercooled water under pressure and the liquid-liquid critical point, J. Chem. Phys. 133(14), 144503 (2010)

39. F. Mallamace, C. Corsaro, and H. E. Stanley, A singular thermodynamically consistent temperature at the origin of the anomalous behavior of liquid water, Sci. Rep. 2, 993 (2012)

40. F. Mallamace, C. Corsaro, D. Mallamace, C. Vasi, and H. E. Stanley, The thermodynamical response functions and the origin of the anomalous behavior of liquid water, Farad. Disc. 167, 95 (2013)

41. J. H. Simpson and H. Y. Carr, Diffusion and nuclear spin relaxation in water, Phys. Rev. 111(5), 1201 (1958)

42. A. Barth and C. Zscherp, What vibrations tell us about proteins, Q. Rev. Biophys. 35(4), 369 (2002)

43. J. T. Pelton and L. R. McLean, Spectroscopic methods for analysis of protein secondary structure, Anal. Biochem. $277(2), 167$ (2000)

44. G. E. Walrafen, M. R. Fisher, M. S. Hokmabadi, and W. H. Yang, Temperature dependence of the low- and highfrequency Raman scattering from liquid water, J. Chem. Phys. 85(12), 6970 (1986)

45. S. Adams, A. M. Higgins, and R. A. L. Jones, Surfacemediated folding and misfolding of proteins at lipid/water interfaces, Langmuir 18(12), 4854 (2002)

46. E. S. Eberhardt and R. T. Raines, Amide-amide and amidewater hydrogen bonds: Implications for protein folding and stability, J. Am. Chem. Soc. 116(5), 2149 (1994) 
47. S. T. R. Walsh, R. P. Cheng, W. W. Wright, D. O. V. Alonso, V. Daggett, J. M. Vanderkooi, and W. F. DeGrado, The hydration of amides in helices; a comprehensive picture from molecular dynamics, IR, and NMR, Protein Sci. 12(3), 520 (2003)

48. M. Sundaralingam and Y. C. Serkharudu, Water-inserted alpha-helical segments implicate reverse turns as folding intermediates, Science 244(4910), 1333 (1989)

49. R. Gilmanshin, S. Williams, R. H. Callender, W. H. Woodruff, and R. B. Dyer, Fast events in protein folding: Relaxation dynamics of secondary and tertiary structure in native apomyoglobin, Proc. Natl. Acad. Sci. USA 94(8), 3709 (1997)

50. F. Mallamace, S. H. Chen, M. Broccio, C. Corsaro, V. Crupi,
D. Majolino, V. Venuti, P. Baglioni, E. Fratini, C. Vannucci, and H. E. Stanley, Role of the solvent in the dynamical transitions of proteins: The case of the lysozyme-water system, J. Chem. Phys. 127(4), 045104 (2007)

51. O. F. A. Larsen and S. Woutersen, Vibrational relaxation of the $\mathrm{H}_{2} \mathrm{O}$ bending mode in liquid water, J. Chem. Phys. 121(24), 12143 (2004)

52. M. G. Sceats and S. A. Rice, In: F. Franks (Ed.), Water - a Comprehensive Treatise, New York: Plenum Press, pp 83-214, 1982

53. M. D. Joesten and L. J. Schaad, Hydrogen Bonding, New York: Marcel Dekker, 1985

54. F. Mallamace, et al., to be published 